\title{
270-W 15-kHz MOPA System Based on Side-pumped Rod-type Nd:YAG Gain Modules
}

\author{
Yong-Ho Cha*, Myoung-Yerl Yang, Kwang-Hoon Ko, Gwon Lim, Jae-Min Han, \\ Hyun-Min Park, Taek-Soo Kim, Sipyo Roh, and Do-Young Jeong \\ Quantum Optics Division, Korea Atomic Energy Research Institute, Daejeon 305-353, Korea
}

(Received October 24, 2008 : revised November 21, 2008 : accepted November 25, 2008)

\begin{abstract}
We have developed a 270-W 15-kHz MOPA system based on side-pumped rod-type Nd:YAG gain modules. The master oscillator is a $3-\mathrm{W} 15-\mathrm{kHz} \mathrm{TEM}_{00} \mathrm{Nd}: \mathrm{YVO}_{4}$ laser with a pulse duration of 30 ns. To preserve the high beam quality during the amplification, we use image relay and polarization rotation which can simultaneously compensate for thermal lensing and thermal birefringence generated in the rod-type gain modules. After the amplification to $270 \mathrm{~W}$ with six rod-type gain modules, the beam quality factor $\left(\mathrm{M}^{2}\right)$ of the amplified laser beam is $5-10$, and the pulse duration is maintained at $30 \mathrm{~ns}$.
\end{abstract}

Keywords: Nd:YAG lasers, High-power lasers, MOPA laser system

OCIS codes : (140.3530) Lasers, neodymium; (140.3280) Laser amplifiers; (140.6810) Thermal effects

\section{INTRODUCTION}

High-average-power high-repetition-rate lasers with a good beam quality are essential tools in many applications such as laser machining, laser-induced EUV generation, and pumping of other solid-state lasers. In particular, laser-based isotope production has been one of the important application fields of high-averagepower high-repetition-rate lasers. Traditionally, laser-based isotope production has been based on dye lasers which can circulate laser media in liquid form and, thus, can reduce the thermal problems generated in a laser medium. Dye lasers, however, have short lifetimes and are difficult to handle and maintain. A new scheme of laser-based isotope production, which was recently proposed for thallium isotopes[1], requires high-power lasers of $\sim 1-\mu$ $\mathrm{m}$ wavelength with a short pulse duration and a high beam quality. In terms of wavelength and power level, a neodymium $(\mathrm{Nd})$-based laser system can be a good candidate for the new laser-based isotope production.

Generally, a high-power high-repetition-rate Nd-based laser system composed of a single oscillator has difficulty in fulfilling the requirements of a short-pulse duration $(<30 \mathrm{~ns})$, a high power $(>200 \mathrm{~W})$, a good beam quality
$\left(\mathrm{M}^{2}<10\right)$, and a high-repetition-rate $(>10 \mathrm{kHz})$ pulsed operation simultaneously. Although some special slabtype lasers can meet such requirements, they are not easy to develop due to their unusual schemes of laser medium and pumping[2,3]. On the other hand, sidepumped rod-type Nd:YAG gain modules are commercially available and widely used[4]. Such rod-type solidstate gain modules, however, have intrinsic problems such as thermal lensing and thermal birefringence which lead to a degradation of beam quality and an increase of depolarization loss[5,6]. Although some schemes have been proposed for the compensation of the thermal birefringence[6-8], it is still challenging to efficiently compensate for the combined effects of the thermal lensing and the thermal birefringence.

In this paper, we report the development of a $270-\mathrm{W}$ $15-\mathrm{kHz}$ MOPA system, ideal for the laser-based production of thallium isotopes, based on rod-type Nd:YAG gain modules. The thermal lensing and the thermal birefringence generated in the gain modules were simultaneously compensated for with a newly proposed scheme [9], and a good beam quality was achieved even after the amplification with six gain modules.

\footnotetext{
*Corresponding author: yhcha@kaeri.re.kr
} 


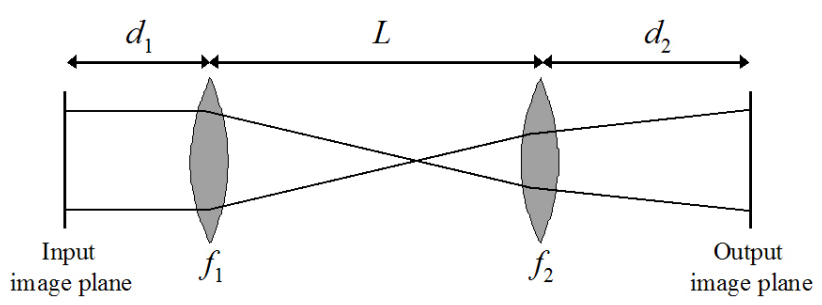

(a)

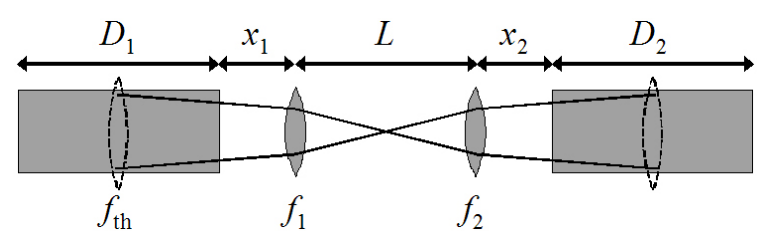

(b)

FIG. 1. (a) Schematic of two-lens image relay. (b) Schematic of the image relay between two rod-type gain modules.

\section{ANALYSIS OF IMAGE RELAY}

The simultaneous compensation of thermal lensing and thermal birefringence can be obtained using the image relay with a pair of positive lenses and the polarization rotation between two identical rods[9]. The image relay with two positive lenses, as shown in Fig. 1, is a wellknown technique[10], but needs to be analyzed with $A B C D$-matrix for the understanding of its unique characteristics. The $A B C D$-matrix of the two-lens image relay can be calculated as follows.

$$
\begin{aligned}
& \left(\begin{array}{ll}
A & B \\
C & D
\end{array}\right)=\left(\begin{array}{ll}
1 & d_{2} \\
0 & 1
\end{array}\right)\left(\begin{array}{cc}
1 & 0 \\
-\frac{1}{f_{2}} & 1
\end{array}\right)\left(\begin{array}{cc}
1 & L \\
0 & 1
\end{array}\right)\left(\begin{array}{cc}
1 & 0 \\
-\frac{1}{f_{1}} & 1
\end{array}\right)\left(\begin{array}{cc}
1 & d_{1} \\
0 & 1
\end{array}\right) \\
& =\left(\begin{array}{cc}
\left(1-\frac{L}{f_{1}}\right)\left(1-\frac{d_{2}}{f_{2}}\right)-\frac{d_{2}}{f_{1}} & L\left(1-\frac{d_{1}}{f_{1}}\right)\left(1-\frac{d_{2}}{f_{2}}\right)+d_{1}\left(1-\frac{d_{2}}{f_{2}}\right)+d_{2}\left(1-\frac{d_{1}}{f_{1}}\right) \\
-\frac{1}{f_{1}}-\frac{1}{f_{2}}-\frac{L}{f_{1}} & \left(1-\frac{L}{f_{2}}\right)\left(1-\frac{d_{1}}{f_{1}}\right)-\frac{d_{1}}{f_{2}}
\end{array}\right)
\end{aligned}
$$

Because the image relay condition is satisfied when $B=0$, the image relay condition is

$$
L\left(1-\frac{d_{1}}{f_{1}}\right)\left(1-\frac{d_{2}}{f_{2}}\right)+d_{1}\left(1-\frac{d_{2}}{f_{2}}\right)+d_{2}\left(1-\frac{d_{1}}{f_{1}}\right)=0
$$

If $L=f_{1}+f_{2}$, above equation becomes a well-known image relay formula in a confocal configuration[10] $(m$ - $\left.d_{1}+d_{2} / m=f_{1}+f_{2}, \quad m=f_{2}+f_{1}\right)$. One can see that the above equation is always satisfied regardless of $L$ if $d_{1}=f_{1}$ and $d_{2}=f_{2}$. This means that the input image at the focus position of the first lens is always relayed to the focus position of the second lens with any separation between two lenses. Therefore, the center of the first rod can be image relayed to the center of the second rod if the following condition is satisfied.

$$
f_{1}=\frac{D_{1}}{2 n}+x_{1}, f_{2}=\frac{D_{2}}{2 n}+x_{2}
$$

where $n$ is the refractive index of the rod, $D_{1}\left(D_{2}\right)$ the length of the first (second) rod, and $x_{1}\left(x_{2}\right)$ the separation between the first (second) rod end and the adjacent lens. If two identical lenses $\left(f_{1}=f_{2}\right)$ and two identical rods $\left(D_{1}=D_{2}\right)$ are used, the image relay condition can be simplified.

The thermal lensing induced in the rod can be independently compensated for if the separation between two lenses $(L)$ is adjusted properly, because the image relay condition of Eq. (3) is independent of $L$. Generally, the output beam from the first rod converges by the thermal lensing effect, and, thus, the input beam to the second rod needs to diverge for the compensation of the second rod's thermal lensing. The magnitudes of the convergence and the divergence of the two rods should be identical for the precise compensation of thermal lensing and thermal birefringence. Such a condition can be found from a lens formula as follows [9].

$$
\frac{1}{f_{t h}}+\frac{L-2 f}{f^{2}}=0
$$

where $f_{\mathrm{th}}$ is the focal length of the thermal lens, and the thermal lens is simplified as a thin lens positioned at the center of the rod. Experimentally, the above condition can be satisfied when the focused point generated between two lenses is positioned exactly at the middle point between two lenses. With the proper image relay, one can compensate for thermal birefringence easily by putting a $90^{\circ}$ quartz rotator between two rods[7].

\section{EXPERIMENTAL COMPENSATION OF THERMAL LENSING AND THERMAL BIREFRINGENCE}

We used two identical rod-type Nd:YAG gain modules to verify the image relay technique described in the previous section. Each gain module had a Nd:YAG rod with a 3 -mm diameter and a $100-\mathrm{mm}$ length, and the rod was side-pumped symmetrically by three laser diode bars. The maximal pumping power for each gain module was $450 \mathrm{~W}$. The single-pass small-signal gain and the thermal focal length of the gain module were measured with respect to the current applied to the laser diode bars. As shown in Fig. 2, the maximal smallsignal gain was 2.3 , and the thermal focal length decreased down to $190 \mathrm{~mm}$ at maximal pumping. The ther- 


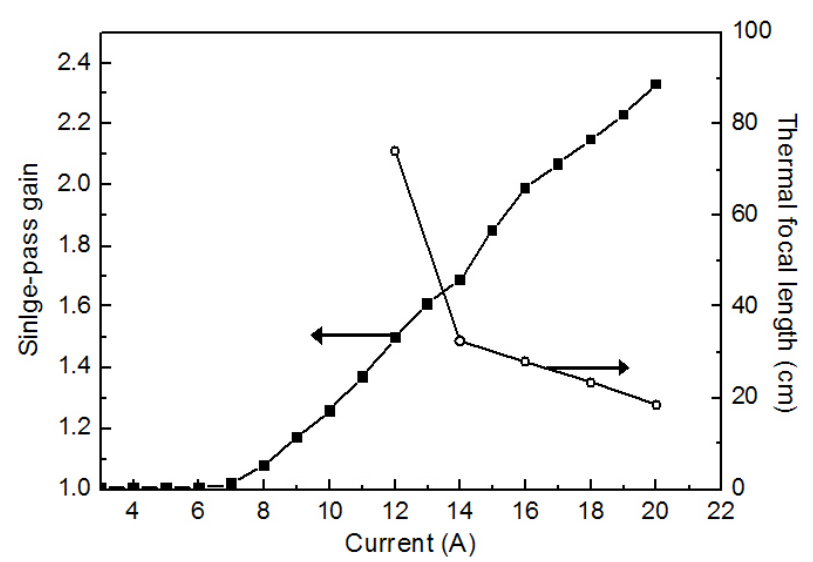

FIG. 2. Small-signal single-pass gain and thermal focal length of the 3 -mm-rod gain module.

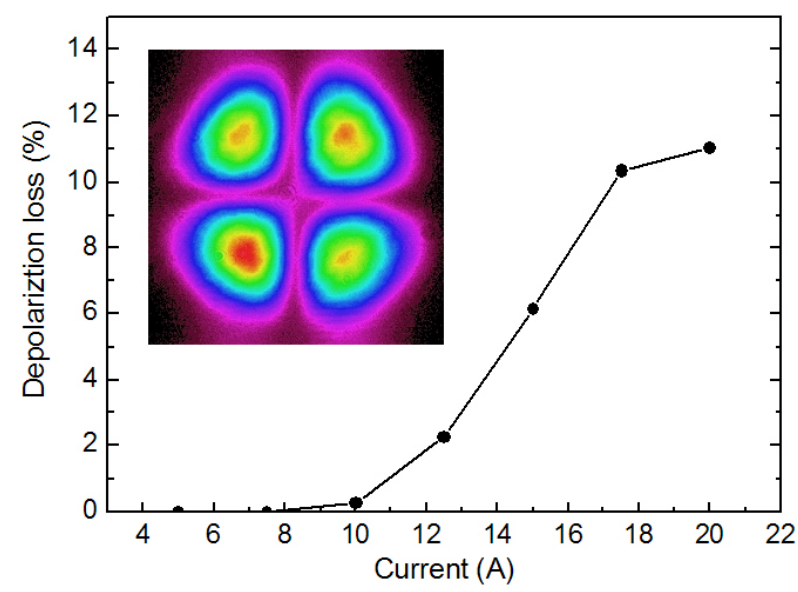

FIG. 3. Single-pass depolarization loss caused by the thermal birefringence in the 3 -mm-rod gain module. The inset is the beam pattern of the depolarized output at the maximal pumping.

mal focal length was measured by finding the position of minimal spot size after the module because very rigorous methods based on wavefront measurement $[11,12]$ are not necessary in our case. Figure 3 shows the depolarization loss of the gain module caused by thermal birefringence. To measure the depolarization loss, we passed a seed laser beam through the gain module and measured the ratio of the depolarized power to the total amplified power as the pumping power increased. As shown in Fig. 3, the maximal depolarization loss was $11 \%$. The inset in Fig. 3 shows the beam pattern of the depolarized output, which is a very typical pattern of thermal-birefringence induced depolarization $[5,6]$.

To compensate for the thermal lensing and the thermal depolarization generated by the gain modules, we used two positive lenses with a 100-mm focal length which is sufficiently shorter than the thermal focal length of the gain module. According to Eq. 3, the separation

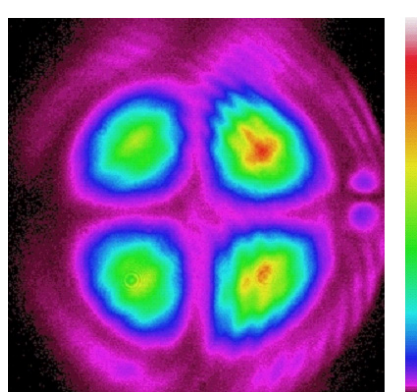

(a)

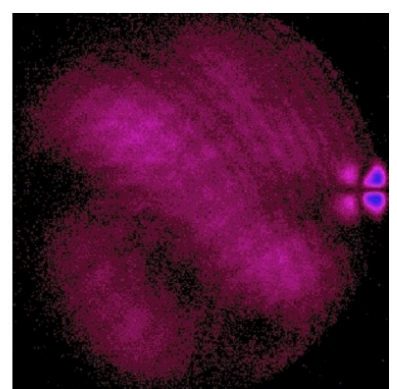

(b)
FIG. 4. Beam pattern of the depolarized output from the two 3-mm-rod gain modules (a) without and (b) with a $90 f$ quartz rotator between the modules.

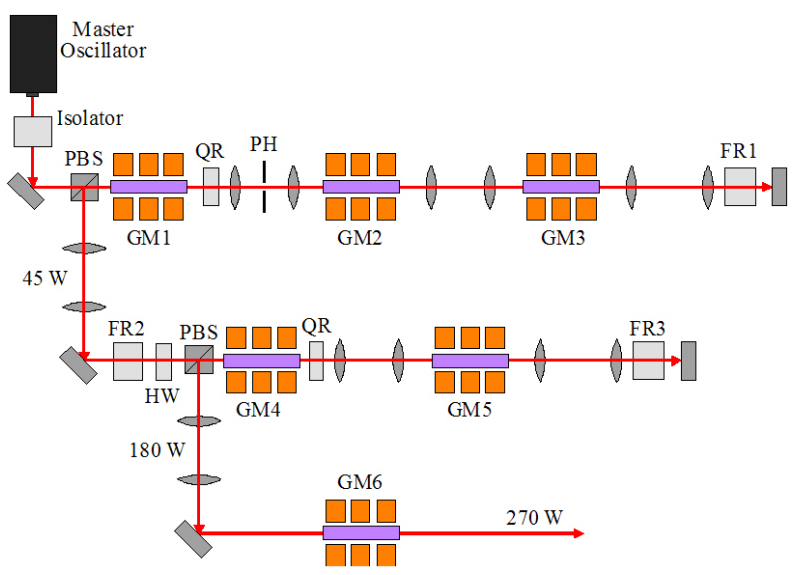

(b)

FIG. 5. Schematic of the 270-W MOPA system. PBS, polarizing beam splitter; GM1-6, Nd:YAG gain modules; QR, $90^{\circ}$ quartz rotator; PH, pinhole; FR1-3, $45^{\circ}$ Faraday rotator; HW, half-wave plate.

between each rod's end and an adjacent lens was set to $72 \mathrm{~mm}$. The distance between the two lenses was adjusted in such a way that the focused point was positioned precisely at the middle point between the two lenses. A $90^{\circ}$ quartz rotator $(\mathrm{QR})$ was placed between two gain modules for the compensation of thermal birefringence. Figure 4 shows the depolarization patterns with and without the quartz rotator. Without the quartz rotator, a very strong depolarized output was generated with a depolarization loss of $21 \%$. With the quartz rotator, on the other hand, the depolarized output was very weak $(\sim 1 \%)$, which shows a very efficient compensation of thermal birefringence.

One drawback of the image relay technique is that the optimal condition of image relay changes depending on the thermal focal length of gain modules. If the thermal focal length changes, the separation between two lenses $(L)$ should be adjusted again according to Eq. (4). Otherwise, the improper image relay can lead to unexpected focusing of laser beam and even damage on 


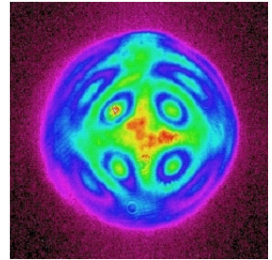

(a)

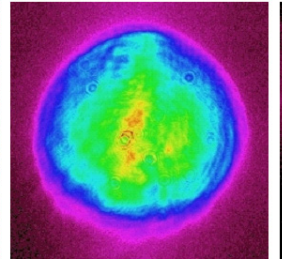

(b)

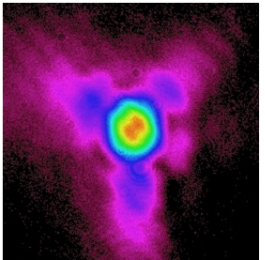

(c)
FIG. 6. Near-field beam pattern after the second AMP (a) without and (b) with thermal birefringence compensation. (c) Far-field beam pattern after the second AMP.

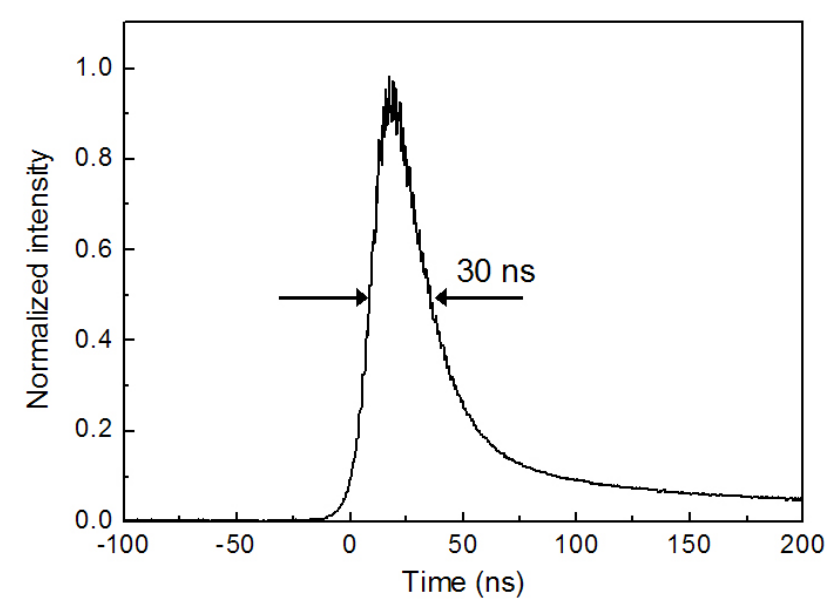

FIG. 7. Temporal profile of an amplified output pulse.

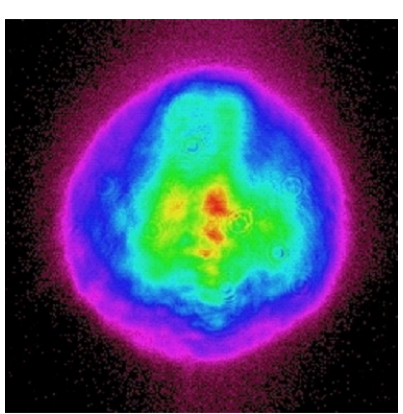

(a)

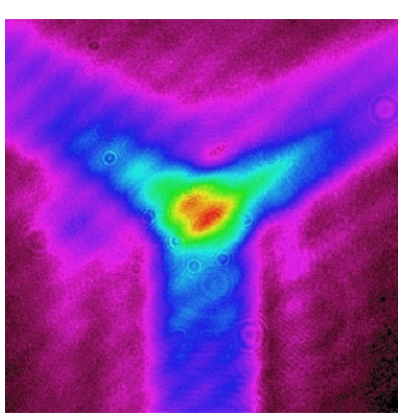

(b)
FIG. 8. (a) Near-field and (b) far-field beam patterns of amplified laser beam.

any optical surface. Therefore, one should fix the pumping power of gain modules at which the image relay is properly designed.

\section{EXPERIMENTAL SETUP AND RESULTS}

The experimental setup of our MOPA system is shown in Fig. 5. The master oscillator was a $15-\mathrm{kHz} 3-\mathrm{W} \mathrm{Nd}$ : $\mathrm{YVO}_{4}$ laser pumped by a fiber-coupled LD. The pulse duration of the master oscillator was $\sim 30$ ns (FWHM) and the spatial mode was $\operatorname{TEM}_{00}\left(\mathrm{M}^{2}<1.2\right)$. For the amplification of the master laser beam, we used three amplifying stages (AMPs). The first AMP was composed of three 3-mm-rod Nd:YAG gain modules (GM1-3) which were described in section III. We installed the gain modules in series and applied the image relay technique between the modules. All the lenses used in the first AMP had a focal length of $100 \mathrm{~mm}$. The amplified power after one pass through the gain modules was $18 \mathrm{~W}$. To extract more power from the gain modules, we made another pass through the modules by retro-reflecting the laser beam. During the retro-reflection, the image of GM3 was relayed to the end mirror first and relayed back to GM3, and we adjusted the separation between image-relaying lenses to make the laser beam wellcollimated at the end mirror. For the compensation of thermal birefringence, we put a QR between GM1 and GM2. The thermal birefringence of GM3 was compensated for with a $45^{\circ}$ Faraday rotator $(\mathrm{FR})$, which gives $90^{\circ}$ polarization rotation after retro-reflection. After double passing, the amplified output was boosted to $45 \mathrm{~W}$.

The second AMP was composed of two 6-mm-rod Nd:YAG gain modules(GM4 and GM5). Each module was side-pumped by a total power of $1.5 \mathrm{~kW}$ from laser diode bars. The laser output from GM1 in the first AMP was image-relayed to GM4 with a magnification factor of 2, and GM4 and GM5 were configured in a similar way to the first AMP. Between the first and the second AMPs, we put a FR and a half-wave plate (HW) to protect the first AMP from any backward laser from the second AMP. The amplified laser power from the second AMP was $180 \mathrm{~W}$. Figure 6(a) and (b) show the near-field beam patterns after the second AMP without and with birefringence compensation, respectively. Without birefringence compensation, the near-field beam pattern shows a strong depolarization pattern, and the depolarization loss was more than $20 \%$ in a double-pass configuration. With birefringence compensation, on the other hand, the depolarization loss was reduced to $\sim 5 \%$, and the near-field beam pattern was significantly improved. The far-field beam pattern (Fig. 6(c)) after the second AMP shows weak and symmetric lobes around a main beam which is caused by non-uniform pumping.

We found that the first and the second AMPs generated self-lasing output when the master laser beam was not seeded into the AMPs. Because the total small-signal gain through the second and the first AMPs in a doublepass configuration is more than 1000, the incomplete isolation between the first and the second AMPs can lead to the oscillation of the depolarized portion. The self-lasing output was $\sim 20 \mathrm{~W}$ without seeding and could be suppressed down to $2 \mathrm{~W}$ with a pinhole installed between GM1 and GM2. The self lasing, on the other hand, was suppressed down completely with the seeding of the master laser beam.

The final AMP was composed of a 9-mm-rod Nd:YAG gain module (GM6) which was pumped by a total power of $2 \mathrm{~kW}$. The laser output from GM4 in the second 
AMP was image-relayed to GM5 with a magnification factor of 1.5. The final AMP was in a single-pass configuration, and the amplified output was $270 \mathrm{~W}$. We could not use double-pass configuration in the final AMP because we had no FR which can be used at a high power of more than $200 \mathrm{~W}$. We expect more than 300 $\mathrm{W}$ of amplified output power if the final AMP is doublepassed with a high power FR. The amplified pulse duration $(\sim 30 \mathrm{~ns})$ was almost the same as that of the master oscillator, as shown in Fig 7. We used a beam profiler to measure the $\mathrm{M}^{2}$ value of the amplified laser beam because the pointing stability of the amplified laser beam $(\sim 0.3 \mathrm{mrad})$ was not good enough to measure the beam size with knife-edge-cutting method. The near-field and the far-field beam patterns are shown in Fig. 8, and the $\mathrm{M}^{2}$ calculated from the near- and far-field beam sizes was 5-10 roughly; the far-field beam size was difficult to determine precisely because the far-field beam pattern has relatively strong lobes.

The conversion efficiency from laser-diode pumping to amplified output of our MOPA system was just $\sim 5 \%$, which is relatively lower than that of other laserdiode pumped Nd:YAG lasers. The main reason for low efficiency is that the laser beam size is sufficiently smaller than the rod diameter of the gain modules. Because a significant overlap of the laser beam with the rod edges leads to a severe degradation of beam quality, we kept the beam size small enough (50-60\% of the rod diameter) during the amplification, and the poor overlap of the laser beam with the active volume of the rods led to the low efficiency.

\section{CONCLUSION}

In conclusion, we have developed a $270-\mathrm{W} 15-\mathrm{kHz}$ MOPA system based on rod-type Nd:YAG gain modules. The thermal lensing and the thermal birefringence generated in the gain modules were simultaneously compensated for by the image relay and the polarization rotation. The rod center of each module was image-relayed to next module's center with two positive lenses, and the separation between two lenses was adjusted to compensate for the thermal lensing. The $90^{\circ}$ polarization rotation between two identical modules decreased the depolarization loss caused by the thermal birefringence significantly. The master oscillator's 3 -W laser output was amplified to $270 \mathrm{~W}$ with six gain modules, and the $\mathrm{M}^{2}$ of the amplified laser beam was 5-10. The pulse duration of 30 ns was maintained during the amplification. Such a high-power MOPA system with a short pulse duration and a good beam quality is ideal for the laser- based production of thallium isotopes.

\section{REFERENCES}

[1] D. Y. Jeong, K. H. Ko, G. Lim, and C. J. Kim, "Method for isotope separation of thallium," US Patent 7,323,651 B2, 2008.

[2] S. Palese, J. Harkenrider, W. Long, F. Chui, D. Hoffmaster, W. Burt, H. Injeyan, G. Conway, G. Truong, and F. Tapos, "High Brightness, End-Pumped, Conduction Cooled Nd:YAG Zig-Zag Slab Laser Architecture," Advanced Solid State Lasers, C. Marshall, ed., vol. 50 of OSA Trends in Optics and Photonics (Optical Society of America, 2001), pp. 41-46.

[3] P. Zhu, D. Li, P. Hu, A. Schell, P. Shi, C. R. Haas, N. Wu, and K. Du, "High efficiency $165 \mathrm{~W}$ near-diffractionlimited Nd:YVO4 slab oscillator pumped at $880 \mathrm{~nm}$," Opt. Lett. vol. 33, pp. 1930-1932, 2008.

[4] H. J. Moon, S. M. Lee, H. S. Kim, D. K. Ko, B. H. Cha, J. H. Yi, and J. M. Lee, "Operation Characteristics of Diode-Pumped, Bifocusing Compensated Two-Rod Nd:YAG Lasers with Diffusive Reflectors," J. Korean Phys. Soc. vol. 39, pp. 881-885, 2001.

[5] W. Koechner and D. K. Rice, "Effect of Birefringence on the Performance of Linearly Polarized YAG:Nd Lasers," IEEE J. Quantum Elec., vol. 6, pp. 557-566, 1970.

[6] J. R. Park, J. Y. Lee, H. S. Kim, K. Y. Um, and H. J. Kong, "Characteristics of a Birefringence Compensation Scheme in Nd3+:YAG Rods Using a Polarization Rotator and Imaging Optics," Opt. Rev. vol. 4, pp. 170175, 1997.

[7] W. C. Scott and M. de Wit, "Birefringence compensation and TEM00 mode enhancement in a Nd:YAG laser," Appl. Phys. Lett. vol. 18, pp. 3-4, 1971.

[8] J. Richards, "Birefringence compensation in polarization coupled lasers," Appl. Opt. vol. 26, pp. 2514-2517, 1987.

[9] R. Bhushan, K. Tsubakimoto, H. Yoshida, H. Fujita, and M. Nakatsuka, "Simultaneous compensation of birefringence and thermal lensing effect in a high average power Nd:YAG amplifier," CLEO/Pacific Rim 2007, Technical Digest WP-11, pp. 557-558.

[10] J. T. Hunt, J. A. Glaze, W. W. Simmons, and P. A. Renard, "Suppression of self-focusing through low-pass spatial filtering and relay imaging," Appl. Opt. vol. 17, pp. 2053-2057, 1978.

[11] T. M. Jeong, C. W. Lee, D. K. Ko, J. M. Lee, and H. S. Kim, "Analysis of a Laser Resonator from the Measurement of Thermal Aberrations in a Laser-Diode Side-Pumped Nd:YAG Laser with a Wavefront Sensor," J. Korean Phys. Soc. vol. 51, pp. 78-83, 2007.

[12] T. M. Jeong, C. M. Kim , D. K. Ko, and J. M. Lee, "Reconstruction of Wavefront Aberration of 100-TW Ti:sapphire Laser Pulse Using Phase Retrieval Method," J. Opt. Soc. Korea, vol. 12, pp. 186-191, 2008. 